\title{
Pan-Mutant-IDH1 Inhibitor Bay-1436032
}

National Cancer Institute

\section{Source}

National Cancer Institute. Pan-Mutant-IDH1 Inhibitor Bay-1436032. NCI Thesaurus.

Code C131690.

An orally available pan-inhibitor of mutant forms of the metabolic enzyme isocitrate dehydrogenase type 1 (IDH1; IDH-1; IDH1 [NADP+] soluble), including forms with mutations of arginine 132 (IDH1(R132)), with potential antineoplastic activity. Upon administration, pan-mutant-IDH-1 inhibitor BAY-1436032 specifically inhibits the activity of IDH1 mutant forms, which prevents the formation of the oncometabolite 2-

hydroxyg lutarate (2HG) from alpha-ketoglutarate (a-KG). This prevents 2 HG-mediated signaling and leads to both an induction of cellular differentiation and an inhibition of cellular proliferation in tumor cells expressing IDH1 mutant forms. IDH1 mutations, including IDH1(R132) mutations, are highly expressed in certain malignancies; they initiate and drive cancer growth by both blocking cell differentiation and catalyzing the formation of $2 \mathrm{HG}$. 